This manuscript is a preprint and has not undergone peer review. Please do not hesitate to contact any of the authors. We welcome any feedback or requests for data. 


\title{
Red noise in steady-state multiphase flow
}

\author{
Catherine Spurin,* Maja Rücker, Marcel Moura, Tom Bultreys, \\ Gaetano Garfi, Steffen Berg, Martin J. Blunt, and Samuel Krevor
}

\begin{abstract}
Understanding the interaction between competing fluids in the pore space of rocks is key for predicting subsurface flow and trapping, such as with $\mathrm{CO}_{2}$ in a saline aquifer. These processes occur over a large span of timescales (from seconds to thousands of years), and length scales (from microns to kilometres). Understanding the link between these temporal and spatial scales will enable us to interpolate between observations made at different resolutions. In this work we explore the temporal scales present during macroscopically steady-state multiphase flow in a porous rock using differential pressure measurements. We observe a cascade of timescales in the pressure differential i.e. a continuous range of frequencies, with lower frequencies having greater amplitudes. We demonstrate a scaling of the spectral density with frequency of $S \sim 1 / f^{2}$, or red noise, to describe the dynamics. This scaling is independent of the flow rate of the fluids or the fraction of the flow taken by water. This red, or Brownian, noise indicates a stochastic process where pressure fluctuations are seen throughout the pore space, resulting in intermittent filling of pores over a wide range of time-scales, from seconds to minutes in these experiments. This observation will aid future modelling of subsurface flow as it suggests self-organised critically of the system with no characteristic time or length scale.
\end{abstract}

\footnotetext{
* cspurin@stanford.edu
} 
The flow of multiple fluids through a rock is a dynamic process involving fluid-fluidrock interactions over multiple spatial and temporal scales. For instance, to understand the trapping of $\mathrm{CO}_{2}$ underground, research spans from the sub mm-scale of high-resolution Xray imaging experiments to observe pore scale phenomena [1-5], the $\mathrm{cm}$-scale of traditional core flooding experiments [6-9], to the km-scale monitoring of $\mathrm{CO}_{2}$ plumes [10-13]. The trapping of $\mathrm{CO}_{2}$, also spans a large range of timescales, from seconds to thousands of years $[14,15]$. All timescales are important for the security of the stored $\mathrm{CO}_{2}[14,16]$. Laboratory experiments provide a wealth of insight into the dynamics. However, these experiments are limited in size and duration. To understand the interaction between spatial and temporal scales, it it is necessary to upscale observations made in the laboratory to the field scale.

The observation that the dynamics of flow in porous media involves phenomena that occur over a wide range of times and distances has necessitated research to bridge these scales [17-23]. But with many flow regimes possible at the pore scale, ranging from ganglion dynamics [24, 25], intermittent pathway flow [26-28], and connected pathway flow (with flow regimes able to exist simultaneously in the pore space [29]), and the heterogeneous nature of most pore spaces of interest, research conducted at the pore scale has often been analysed independently of the impact on larger scale flow. As a result, the dynamics at the pore scale are overlooked in the framework of continuum scale models. However, the pore scale dynamics may play a role in larger scale flow properties, and will influence plume migration $[17,26,30]$.

Typically, the pressure measured during steady-state, which is used to calculate the relative permeability of the fluid phases, shows notable fluctuations $[3,25,26,28,29,31-$ 35]. Historically these fluctuations have been discounted as noise or a failed experiment, such that there is extensive literature on steady-state relative permeability, but in most cases the raw pressure data is not shown. Recent research has proposed that these pressure fluctuations that are typically disregarded are actually capillary effects [36]. This suggests that the fluctuations present in the pressure data may provide the necessary link between flow processes across scales.

A power spectra analysis of the pressure data has been conducted for drainage experiments in a quasi-2D porous medium; the power spectral density, $S$, of the differential pressure measured across the sample were related to the frequency domain, $f[37,38]$. Different exponents, $\alpha$, were observed for different frequency ranges, where 


$$
S(f) \sim \frac{1}{f^{\alpha}}
$$

with $1 \leq \alpha \leq 3.5$. This dynamic scaling requires different analytical models to represent the data. For drainage experiments in 3D porous media, a stretched exponential was used to model the power spectral density in pressure data instead of a simple power law [39]:

$$
\log S(f) \sim f^{\frac{1}{2}}
$$

This was linked to the multiscale heterogeneity of the system, and was also observable during droplet coalescence [39].

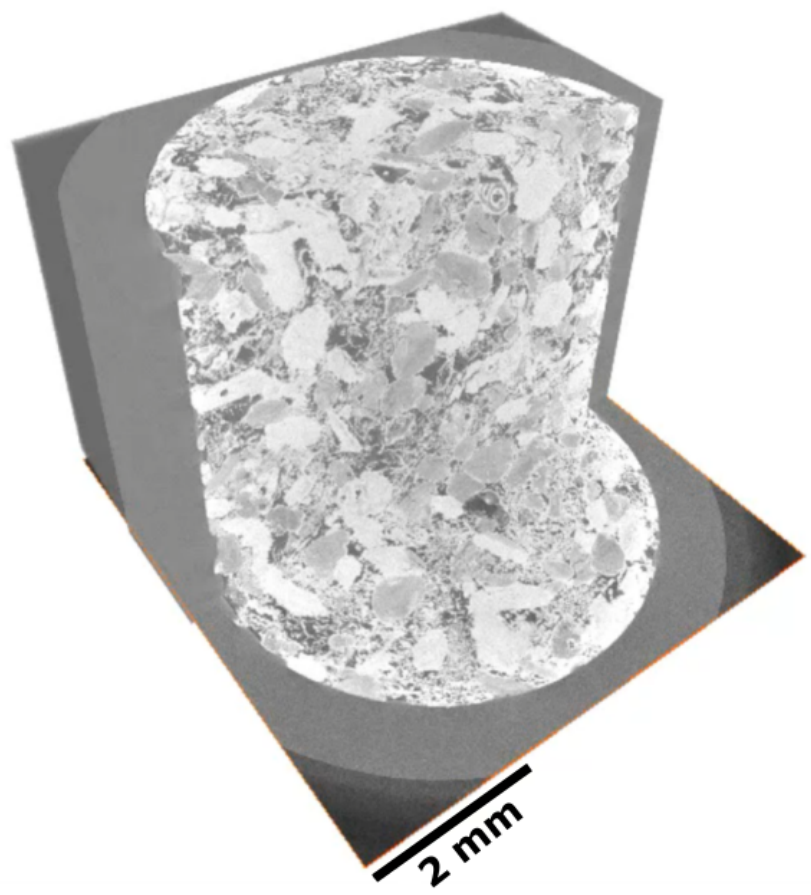

FIG. 1: Pore space of Estaillades carbonate rock sample used in these experiments. The sample was $5 \mathrm{~mm}$ in diameter and $21 \mathrm{~mm}$ in length.

While these findings are of great interest, they were only found prior to breakthrough of the invading fluid, and may not be applicable to steady-state processes, when the averaged saturation remains constant. Steady-state or quasi-steady-state conditions are important in process that occur over long time-scales, such as carbon dioxide and hydrogen storage, 
as well as the continued operation of processes in manufactured materials, such as water transport in the gas diffusion layer of a fuel cell [40-44].

In this work we will show that the pressure fluctuations recorded during steady-state flow can be linked to the dynamics in the pore space. We will conduct a power spectra analysis for different flow rates, during capillary dominated steady-state flow.

We recorded the differential pressure across a heterogeneous carbonate sample (shown in Figure 1) during steady-state flow of nitrogen gas and brine. Nitrogen gas and brine were injected simultaneously through a porous rock at three total flow rates: $0.1 \mathrm{ml} / \mathrm{min}$, $0.4 \mathrm{ml} / \mathrm{min}$ and $0.7 \mathrm{ml} / \mathrm{min}$. Within each total flow rate, the proportion constituted by the brine flow, referred to as the fractional flow $f_{w}$, was varied three times: $0.85,0.7$ and 0.5. This leads to a total of 9 experiments, as listed in Table I. The capillary number is calculated using

$$
C a=\frac{q}{\sigma \lambda}
$$

where $q$ is the total Darcy flux (volume of both water and gas injected per unit area per unit time), $\sigma$ is the interfacial tension between gas and water $\left(47 \times 10^{-3} \mathrm{~N} / \mathrm{m}\right)$, and $\lambda$ is the mobility ratio, as defined in [2]:

$$
\lambda=\frac{f_{w}}{\mu_{w}}+\frac{1-f_{w}}{\mu_{g}},
$$

where $\mu_{w}$ is the viscosity of the water $\left(85.3 \times 10^{-5} \mathrm{~Pa} \cdot \mathrm{s}\right)$, and $\mu_{g}$ is the viscosity of the gas $\left(2.08 \times 10^{-5} \mathrm{~Pa} \cdot \mathrm{s}\right)$. The capillary numbers are listed in Table I; they show that the flow was capillary-dominated.

The pressure was measured across the rock at the inlet and outlet of the flow lines every $128 \mathrm{~ms}$ over a period of $1 \mathrm{hr}$. These pressure recordings during steady-state flow (where the pressure drop is constant when averaged over time) are shown in Figure 2.

For experiments 1-3 in Figure 2, there is a clear cycle in the pressure data, with a period of approximately 10 minutes. From the X-ray images acquired during these experiments, 10 minutes corresponds to the longest time frame also observed for changes in pore-scale fluid occupancy [26]. However, other timescales are observed in the X-ray images. The methodology of the X-ray imaging is described elsewhere [26, 45]. A subvolume of the pore space during experiment 1 is shown in Figure 3. This is to highlight the range of timescales 


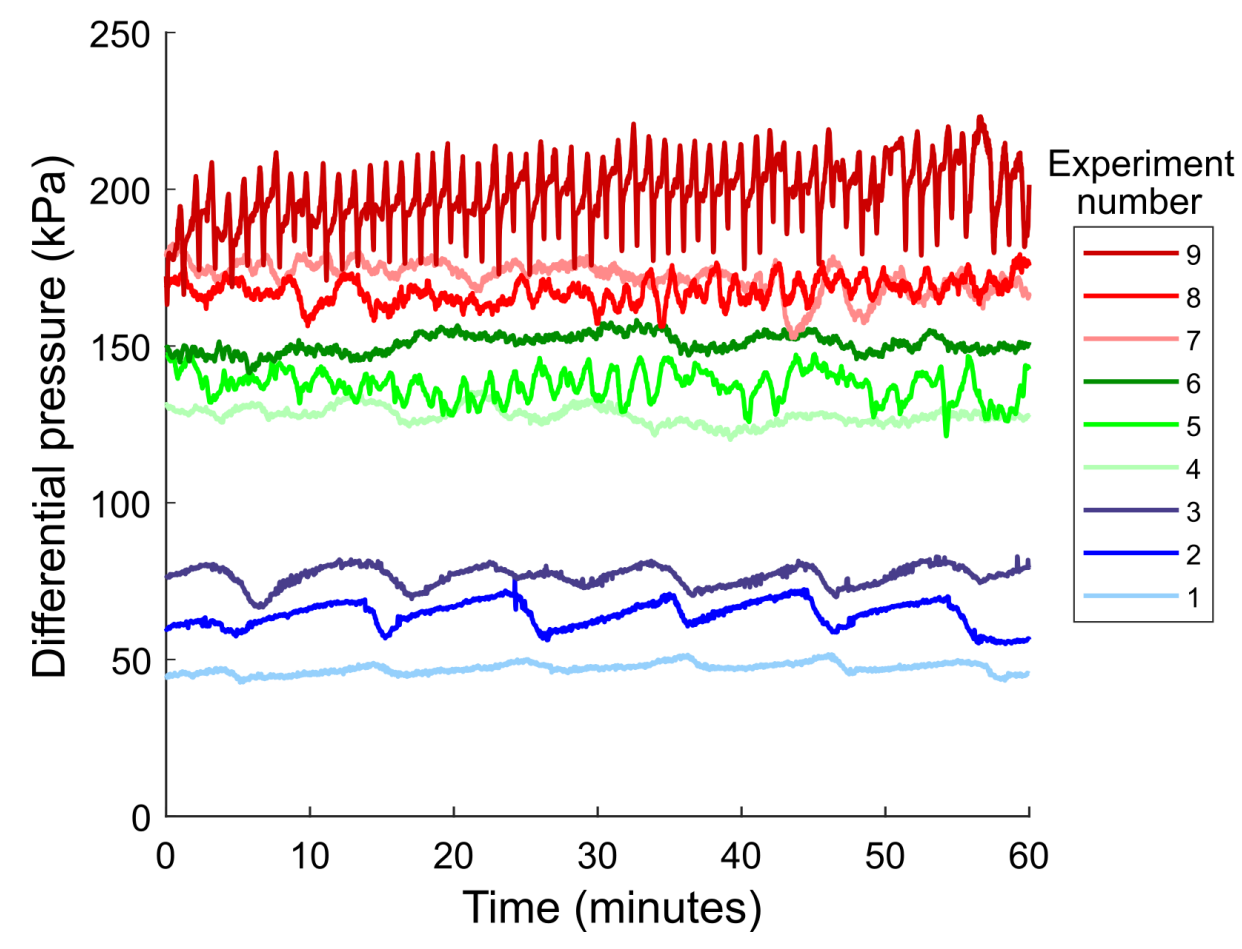

FIG. 2: Differential pressure data for an hour during steady-state for all flow experiments. The experimental parameters are listed in Table I.

observed qualitatively in the pore space.

For this subvolume we easily identify three significantly different timescales:

1. Panel 1: a ganglion of gas repeatedly connects and disconnects. Without this connection (indicated by the arrow), the gas is completely disconnected from the flow across the sample. The connection repeats approximately every 100 scans, or 8-10 mins.

2. Panel 2: while the gas ganglion is disconnected, the gas interface that is connected to the main flow oscillates without reconnecting to the ganglion. This intermittent pore does not influence the connectivity but may influence energy dissipation. This intermittent gas occupancy repeats approximately every 30 scans, or 1-2 mins.

3. Panel 3: while the gas ganglion is connected, the gas interface indicated by the arrow intermittently fluctuates. This intermittent gas occupancy repeats approximately every 15 scans, or every 30 second to 1 min.

More subtly, the connection between the main flow and the ganglion changes in width 
TABLE I: Experimental parameters.

\begin{tabular}{|l|l|l|l|}
\hline $\begin{array}{c}\text { Experiment } \\
\text { number }\end{array}$ & $\begin{array}{c}\text { Total flow } \\
\text { rate } \\
(\mathrm{ml} / \mathrm{min})\end{array}$ & $\begin{array}{c}\text { Fractional } \\
\text { flow } \\
\left(f_{w}\right)\end{array}$ & $\begin{array}{c}\text { Capillary } \\
\text { number }\end{array}$ \\
\hline 1 & 0.1 & 0.85 & $16.2 \times 10^{-8}$ \\
\hline 2 & 0.1 & 0.7 & $8.7 \times 10^{-8}$ \\
\hline 3 & 0.1 & 0.5 & $5.4 \times 10^{-8}$ \\
\hline 4 & 0.4 & 0.85 & $6.5 \times 10^{-7}$ \\
\hline 5 & 0.4 & 0.7 & $3.5 \times 10^{-7}$ \\
\hline 6 & 0.4 & 0.5 & $2.1 \times 10^{-7}$ \\
\hline 7 & 0.7 & 0.85 & $11.4 \times 10^{-7}$ \\
\hline 8 & 0.7 & 0.7 & $6.1 \times 10^{-7}$ \\
\hline 9 & 0.7 & 0.5 & $3.8 \times 10^{-7}$ \\
\hline
\end{tabular}

in panel 3 of Figure 3, on the order of 10-20 microns. This again, does not influence the connectivity of the gas but may play an important role in energy dissipation and flow conductance.

To explore the time scales quantitatively in the pressure data, we transform the pressure data from the time domain into the frequency domain using a Fourier transform, with the power spectrum for each total flow rate shown in Figure 4. A cascade of temporal scales is observable.

The highest amplitudes for the lower frequencies in Figure 4 correspond to timescales of 810 minutes. In these experiments, intermittent pathway flow was observed, with the longest timescale corresponding to the filling repetitions in the largest intermittently occupied pores [26]. The generation of intermittency in pores is linked to the onset of inertial forces at key locations in the pore space [45]. This suggests the timescales observed in the pressure data are induced through inertial forces, and energy is dissipated at all length scales from the maximum intermittent pore.

The highest frequencies correspond to the recording interval of the pressure transducer. However, the imaging interval for the experiments was 1 second. As there was no blurring in the images, it was concluded that the minimum timescale was greater than 1 second. This 


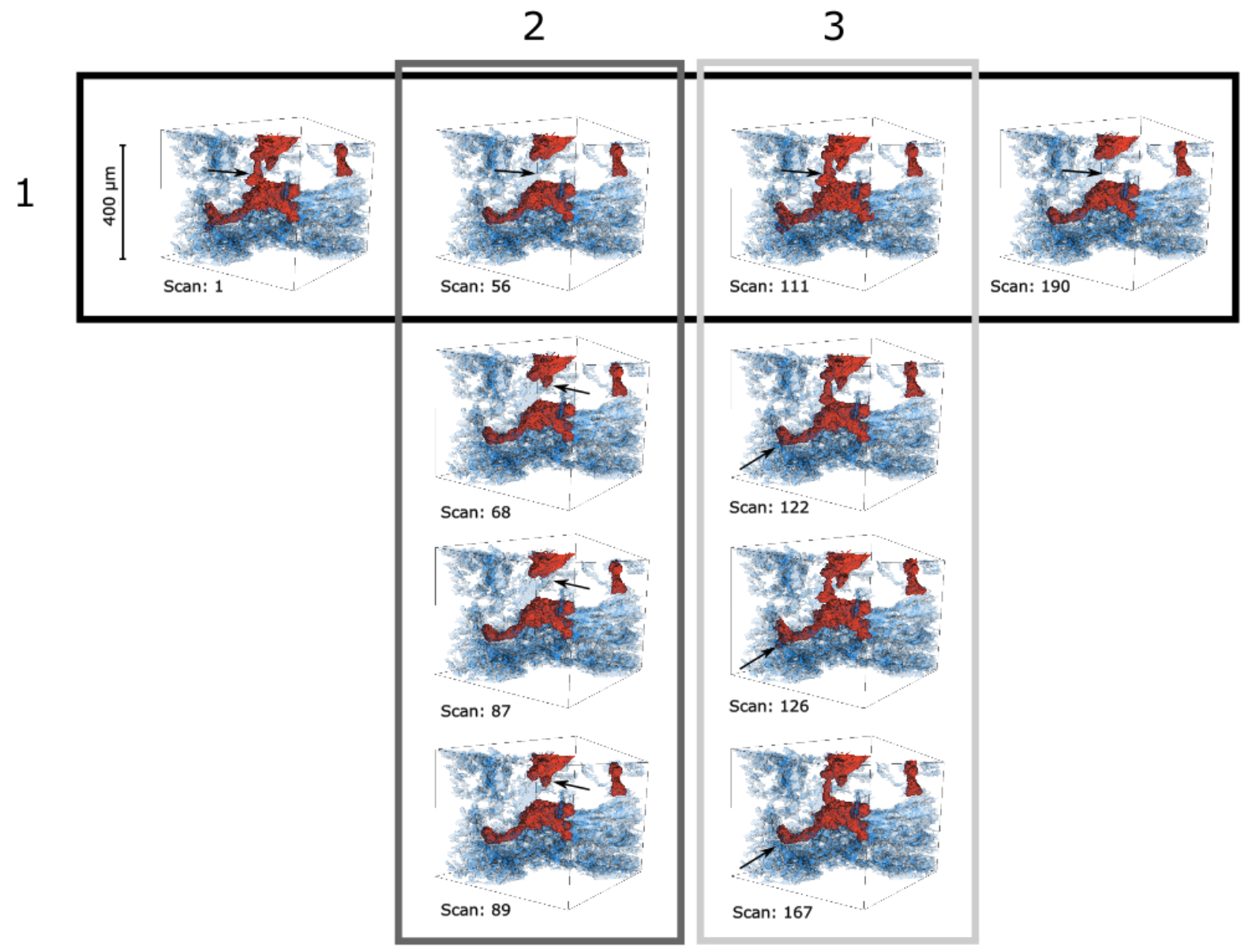

FIG. 3: Timescales present in intermittent gas occupancy. Nitrogen is shown in red, brine in blue and the rock grains are transparent. We observe a gas ganglion repeatedly connect and disconnect in the top panel. Other fluctuations, described in the text, are observed in panels 2 and 3, these occur over shorter time frames.

could mean that the Fourier transform for the higher frequencies is either noise, or small dynamics below the resolution of imaging. This is discussed further in the Supplementary Material.

As shown in Figure 4, the power spectral density, $S$, is correlated with the frequency, $f$. A slope with gradient of -2 is plotted in Figure 4. This slope characterises the relationship well, suggesting:

$$
S(f) \sim \frac{1}{f^{2}}
$$




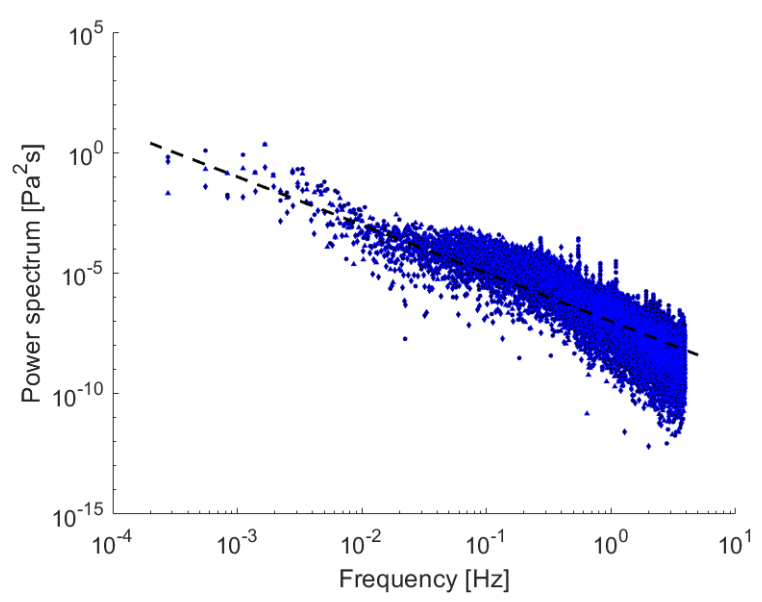

(a)

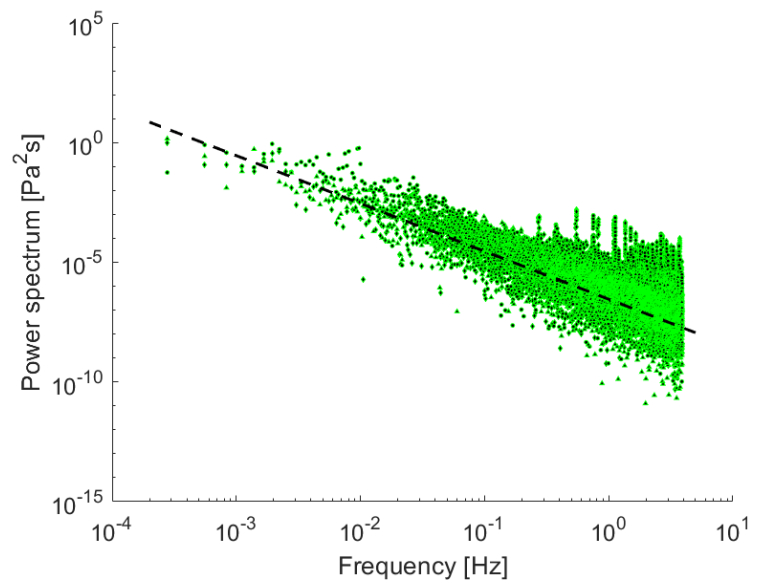

(b)

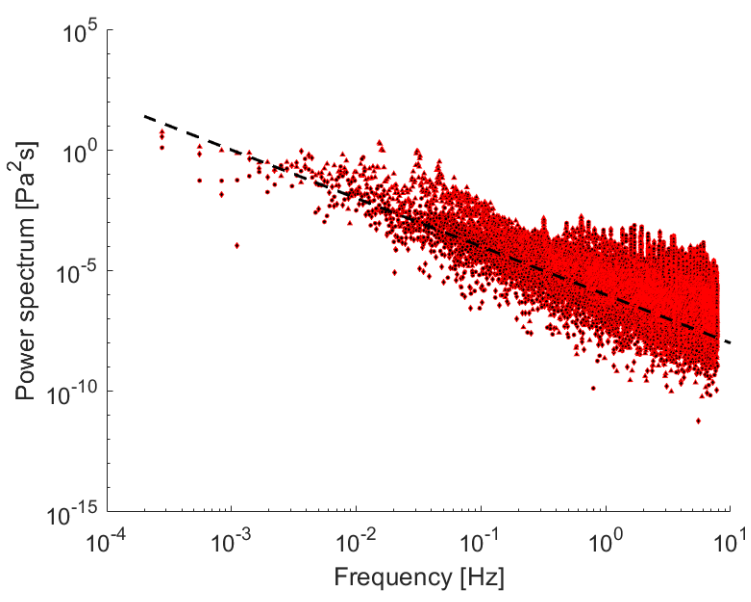

(c)

FIG. 4: Power spectral density for a) total flow rate $=0.1 \mathrm{ml} / \mathrm{min}$ i.e. experiments $1-3, \mathrm{~b}$ ) total flow rate $=0.4 \mathrm{ml} / \mathrm{min}$ i.e. experiments $4-6$, and c) total flow $=$ rate $0.7 \mathrm{ml} / \mathrm{min}$ i.e. experiments 7-9. Each graph shows the results from three experiments for different fractional flows. The black dashed line denotes $1 / f^{2}$ scaling. Experimental parameters listed in Table I.

For steady-state flow, where both drainage and imbibition events are occurring in the pore space, and the saturation remains fairly constant, the scaling is consistent across all frequencies. The scaling is independent of the total pressure drop across the sample which increases by a factor of 4 across the experiments (Figure 2).

Red noise can be represented analytically with a signal composed of a train of exponentially decaying pulses, $p$, located at randomly distributed discrete times, $t_{j}$, 


$$
p(t)=\sum_{j} A H\left(t-t_{j}\right) e^{-\lambda\left(t-t_{j}\right)},
$$

where $\lambda$ and $A$ are the characteristic decay rate and amplitude of the pulses, respectively. They are initially taken to be constants. $H\left(t-t_{j}\right)$ is the Heaviside step function, i.e. $H\left(t-t_{j}\right)=0$ if $t<t_{j}$ and $H\left(t-t_{j}\right)=1$ if $t \geq t_{j}$.

If $P(f)$ be the Fourier transform of $p(t)$ then the power spectral density, $S_{\lambda}(f)$, is

$$
S(f)=\lim _{T \rightarrow \infty} \frac{1}{T}\left\langle|P(f)|^{2}\right\rangle=\frac{A^{2} r}{\lambda^{2}+4 \pi^{2} f^{2}},
$$

where $r$ is the average temporal rate of occurrence of pulses in the signal and the brackets are the expected value operator. Eq. (7) is a Lorentzian curve which is approximately constant for lower frequencies $(f \ll \lambda / 2 \pi)$ and decays as $1 / f^{2}$ for higher frequencies $(f \gg \lambda / 2 \pi)$. If the decay rate $\lambda$ is sufficiently low in comparison with the typical frequencies in the experiment, the constant regime of Eq. (7) may not be visible in the data. In this situation, the $\lambda^{2}$ term in the denominator becomes negligible and Eq. 7 reduces to

$$
S(f)=\frac{A^{2} r}{4 \pi^{2} f^{2}},
$$

which the $1 / f^{2}$ scaling observed in the experiments.

Hierarchical stochastic processes generate $1 / f^{\alpha}$ noise [46, 47], so such a scaling was expected in multiphase flow, as the characteristic times are correlated with event size. Red noise is observed classically in Brownian motion. For multiphase flow in porous media, this implies a stochastic fluctuation of pressure as a random walk throughout the pore space, associated with the intermittent filling of intermediate sized pores. There are similarities between observations made in unsteady-state flow [37, 38], with one scaling fitting the observations made at steady-state. In general, $1 / f^{2}$, or red noise, is observed in many Earth science applications, especially oceanography, where temperature fluctuations are observed over a wide range of spatial and temporal scales [48-50]. Future work is needed to understand the universality of our observations with different rock structures and wettabilities. Furthermore, it would be of great interest to understand if the $1 / f^{2}$ scaling can be derived 
from percolation theory.

[1] C. Spurin, T. Bultreys, B. Bijeljic, M. J. Blunt, and S. Krevor, Physical Review E 100, 043103 (2019).

[2] C. Spurin, T. Bultreys, B. Bijeljic, M. J. Blunt, and S. Krevor, Physical Review E 100, 043115 (2019).

[3] Y. Gao, A. Q. Raeini, M. J. Blunt, and B. Bijeljic, Advances in Water Resources 129, 56 (2019).

[4] M. Andrew, B. Bijeljic, and M. J. Blunt, International Journal of Greenhouse Gas Control 22, 1 (2014).

[5] S. Zou, R. T. Armstrong, J.-Y. Arns, C. Arns, and F. Hussain, Water Resources Research (2018).

[6] S. J. Jackson, S. Agada, C. A. Reynolds, and S. Krevor, Water Resources Research 54, 3139 (2018).

[7] J. Oh, K.-Y. Kim, W. S. Han, M. Kim, and E. Park, Journal of Petroleum Science and Engineering 172, 1174 (2019).

[8] C.-w. Kuo, J.-C. Perrin, S. M. Benson, et al., in SPE Western Regional Meeting (Society of Petroleum Engineers, 2010).

[9] C. A. Reynolds, M. J. Blunt, and S. Krevor, Water Resources Research 54, 729 (2018).

[10] H. Alnes, O. Eiken, S. Nooner, G. Sasagawa, T. Stenvold, and M. Zumberge, Energy Procedia 4, $5504(2011)$.

[11] R. Arts, O. Eiken, A. Chadwick, P. Zweigel, L. Van der Meer, and B. Zinszner, in Greenhouse Gas Control Technologies-6th International Conference (Elsevier, 2003) pp. 347-352.

[12] M. Hesse, H. A. Tchelepi, F. M. Orr, et al., in SPE Annual Technical Conference and Exhibition (Society of Petroleum Engineers, 2006).

[13] J. M. Nordbotten, M. A. Celia, and S. Bachu, Transport in Porous media 58, 339 (2005).

[14] S. M. Benson, K. Bennaceur, P. Cook, J. Davison, H. de Coninck, K. Farhat, C. Ramirez, D. Simbeck, T. Surles, P. Verma, et al., Global energy assessment-Toward a sustainable future , 993 (2012).

[15] E. Rubin and H. De Coninck, UK: Cambridge University Press. TNO (2004): Cost Curves for 
CO2 Storage, Part 2, 14 (2005).

[16] S. Krevor, M. J. Blunt, S. M. Benson, C. H. Pentland, C. Reynolds, A. Al-Menhali, and B. Niu, International Journal of Greenhouse Gas Control 40, 221 (2015).

[17] S. J. Jackson and S. Krevor, Geophysical Research Letters 47, e2020GL088616 (2020).

[18] S. J. Jackson, Q. Lin, and S. Krevor, Water Resources Research 56, e2019WR026396 (2020).

[19] A. H. Kohanpur and A. J. Valocchi, Transport in Porous Media 135, 659 (2020).

[20] M. Rücker, W.-B. Bartels, G. Garfi, M. Shams, T. Bultreys, M. Boone, S. Pieterse, G. Maitland, S. Krevor, V. Cnudde, et al., Journal of colloid and interface science 562, 159 (2020).

[21] M. Rücker, W.-B. Bartels, T. Bultreys, M. Boone, K. Singh, G. Garfi, A. Scanziani, C. Spurin, S. Yesufu-Rufai, S. Krevor, et al., Petrophysics 61, 189 (2020).

[22] S. Zou, R. Armstrong, et al., SPE Journal 24, 1 (2019).

[23] H. Wu, D. Wang, and D. K. Schwartz, The Journal of Physical Chemistry Letters 11, 8825 (2020).

[24] D. Avraam and A. Payatakes, Journal of Fluid Mechanics 293, 207 (1995).

[25] S. S. Datta, T. Ramakrishnan, and D. A. Weitz, Physics of Fluids 26, 022002 (2014).

[26] C. Spurin, T. Bultreys, M. Rücker, G. Garfi, C. M. Schlepütz, V. Novak, S. Berg, M. J. Blunt, and S. Krevor, Water Resources Research 56, e2020WR028287 (2020).

[27] C. A. Reynolds, H. Menke, M. Andrew, M. J. Blunt, and S. Krevor, Proceedings of the National Academy of Sciences 114, 8187 (2017).

[28] Y. Gao, Q. Lin, B. Bijeljic, and M. J. Blunt, Water Resources Research 53, 10274 (2017).

[29] M. Rücker, S. Berg, R. Armstrong, A. Georgiadis, H. Ott, A. Schwing, R. Neiteler, N. Brussee, A. Makurat, L. Leu, et al., Geophysical Research Letters 42, 3888 (2015).

[30] R. Juanes, C. W. MacMinn, and M. L. Szulczewski, Transport in porous media 82, 19 (2010).

[31] H. Menke, Y. Gao, S. Linden, and M. Andrew, (2019).

[32] Y. Gao, Q. Lin, B. Bijeljic, and M. J. Blunt, Physical Review Fluids 5, 013801 (2020).

[33] Z. P. Alcorn, S. Fredriksen, M. Sharma, T. L. Føyen, C. Wergeland, M. Fernø, A. Graue, and G. Ersland, (2020).

[34] Y. Wang and S. K. Masalmeh, in E3S Web of Conferences, Vol. 89 (EDP Sciences, 2019) p. 02007.

[35] Q. Lin, B. Bijeljic, S. Berg, R. Pini, M. J. Blunt, and S. Krevor, Physical Review E 99, 063105 (2019). 
[36] M. Rucker, A. Georgiadis, R. T. Armstrong, H. Ott, N. Brussee, H. Van der Linde, L. Simon, F. Enzmann, M. Kersten, and S. Berg, (2021).

[37] M. Moura, K. J. Måløy, E. G. Flekkøy, and R. Toussaint, Frontiers in Physics 7, 217 (2020).

[38] M. Moura, K. J. Måløy, E. G. Flekkøy, and R. Toussaint, Physical review letters 119, 154503 (2017).

[39] J. E. McClure, S. Berg, and R. T. Armstrong, arXiv preprint arXiv:2012.09139 (2020).

[40] R. Haszeldine, O. Quinn, G. England, M. Wilkinson, Z. Shipton, J. P. Evans, J. Heath, L. Crossey, C. Ballentine, and C. Graham, Oil \& gas science and technology 60, 33 (2005).

[41] C. Ehlig-Economides and M. J. Economides, Journal of Petroleum Science and Engineering 70, $123(2010)$.

[42] A. D. Lueking and R. T. Yang, Applied Catalysis A: General 265, 259 (2004).

[43] V. Kong, D. Kirk, F. Foulkes, and J. Hinatsu, International Journal of Hydrogen Energy 28, 205 (2003).

[44] R. M. Rao, D. Bhattacharyya, R. Rengaswamy, and S. Choudhury, Journal of Power Sources 173, 375 (2007).

[45] C. Spurin, T. Bultreys, M. Rücker, G. Garfi, C. M. Schlepütz, V. Novak, S. Berg, M. J. Blunt, and S. Krevor, Advances in Water Resources 150, 103868 (2021).

[46] A. Steyer, P. Guenoun, and D. Beysens, Physical review letters 68, 1869 (1992).

[47] P. Bak, C. Tang, and K. Wiesenfeld, Physical review A 38, 364 (1988).

[48] J. E. Overland, D. B. Percival, and H. O. Mofjeld, Deep Sea Research Part I: Oceanographic Research Papers 53, 582 (2006).

[49] P. Sura and S. T. Gille, Journal of marine research 61, 313 (2003).

[50] D. L. Rudnick and R. E. Davis, Deep Sea Research Part I: Oceanographic Research Papers 50, 691 (2003). 